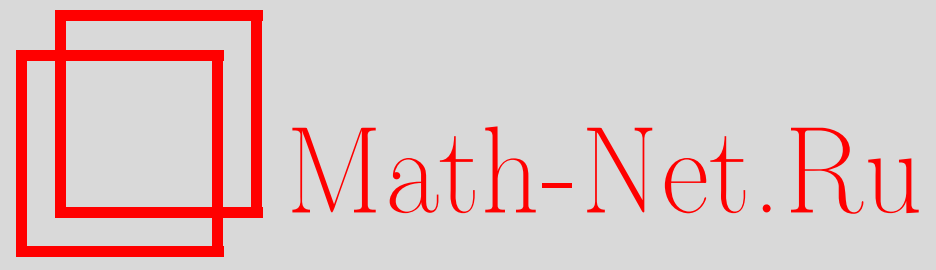

Н. Н. Холщевникова, Сумма всюду сходящегося тригонометрического ряда, Матем. заметки, 2004, том 75, выпуск $3,470-473$

DOI: https://doi.org/10.4213/mzm549

Использование Общероссийского математического портала Math-Net.Ru подразумевает, что вы прочитали и согласны с пользовательским соглашением http://www. mathnet.ru/rus/agreement

Параметры загрузки:

IP : 54.198 .55 .26

26 апреля 2023 г., 07:09:35

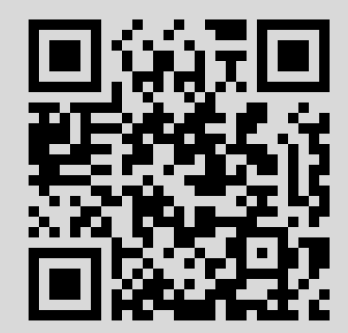




\section{СУММА ВСЮДУ СХОДЯЩЕГОСЯ ТРИГОНОМЕТРИЧЕСКОГО РЯДА}

\section{Н. Н. Холщевникова}

В данной работе рассматриваются два вопроса, касающиеся суммы всюду сходящегося тригонометрического ряда. Хорошо известна (см., например, [1, гл. I, § 72])

ТЕОРЕМА ДЮ БУА-РЕЙМОНА-ЛЕБЕГА. Если тригонометрический ряд

$$
\frac{a_{0}}{2}+\sum_{n=1}^{\infty} a_{n} \cos n x+b_{n} \sin n x
$$

всюду на $[0,2 \pi)$ сходится $к$ ограниченной функиии $f$, то этот ряд есть ряд Фурье функu,u $f$.

ЗАмЕч АнИЕ. Из доказательства теоремы следует, что ее утверждение остается справедливым, если ряд (1) с условием

$$
\lim _{n \rightarrow \infty} a_{n}=\lim _{n \rightarrow \infty} b_{n}=0
$$

всюду суммируется к функции $f$ методом Римана.

Эта теорема была доказана дю Буа-Реймоном [2] для ограниченной, интегрируемой по Риману функции $f$ в 1876 г. и обобщена Лебегом [3] на случай ограниченной (и, автоматически, интегрируемой по Лебегу) функции в 1903 г.

На вопрос Б. С. Кашина о примере тригонометрического ряда всюду сходящегося к ограниченной, но не интегрируемой по Риману функции, автор не только сразу не ответил, но и не нашел соответствуюшего примера в широко известной литературе по тригонометрическим рядам [1], [3]-[6] (что естественно не исключает наличие такого примерав других опубликованных или нет работах). Приведем здесь (упрощенный после обсуждения с В. А. Скворцовым)

ПРИМЕР ограниченной, но не интегрируемой по Риману функции, являющейся суммой всюду сходящегося тригонометрического ряда.

Пусть $E$ - совершенное, нигде не плотное множество положительной меры на $[0,2 \pi)$, например, канторовское множество положительной меры. Занумеруем каким-нибудь способом смежные интервалы множества $E$ в последовательность $I_{i}=\left(a_{i}, b_{i}\right), i \in \mathbb{N}$. Для каждого интервала $I_{i}$ через $J_{i}$ обозначим подотрезок

$$
J_{i}=\left[\frac{a_{i}+b_{i}}{2}-\frac{b_{i}-a_{i}}{4^{i}}, \frac{a_{i}+b_{i}}{2}+\frac{b_{i}-a_{i}}{4^{i}}\right] .
$$

Работа выполнена при поддержке Российского фонда фундаментальных исследований, грант № 02-01-00428. 
Обозначим через $f_{i}$ функцию, равную нулю вне $J_{i}$ и на концах отрезка $J_{i}$, единице в середине $J_{i}$ и линейную в левой и правой половинах отрезка $J_{i}$. Функции $f_{i}, i \in \mathbb{N}$, непрерывны на $[0,2 \pi)$. Положим

$$
f(x)=\sum_{i=1}^{\infty} f_{i}(x), \quad x \in[0,2 \pi) .
$$

Таким образом,

$$
f(x)= \begin{cases}f_{i}(x), & x \in I_{i}(i \in \mathbb{N}), \\ 0, & x \in E\end{cases}
$$

Очевидно, что $f$ - ограниченная функция первого класса Бэра, $f$ непрерывна на смежных интервалах $I_{i}, i \in \mathbb{N}$, и разрывна в каждой точке множества $E$. Следовательно, $f$ не интегрируема по Риману в силу критерия Лебега, так как множество ее точек разрыва имеет положительную меру. Ряды Фурье функций $f_{i}, i \in \mathbb{N}$, равномерно сходятся к $f_{i}$ на $[0,2 \pi)$. В силу принципа локализации Римана отсюда и из определения функции $f$ следует, что ее ряд Фурье $S(f)$ сходится к $f$ на каждом из смежных интервалов $I_{i}, i \in \mathbb{N}$. Осталось показать, что $S(f)$ сходится к нулю на $E$.

Пусть $x \in E, \delta>0$. Положим

$$
A(x, \delta)=\left\{i \in \mathbb{N}: I_{i} \cap(x, x+\delta) \neq \varnothing\right\}, \quad B(x, \delta)=\left\{i \in \mathbb{N}: I_{i} \cap(x-\delta, x) \neq \varnothing\right\},
$$

и оценим интегралы $\int_{x}^{x+\delta} f(t) \frac{\sin n(t-x)}{t-x} d t$ и $\int_{x-\delta}^{x} f(t) \frac{\sin n(t-x)}{t-x} d t$.

Имеем

$$
\begin{aligned}
& \left|\int_{x}^{x+\delta} f(t) \frac{\sin n(t-x)}{t-x} d t\right| \leqslant \sum_{i \in A(x, \delta)} \int_{J_{i}} \frac{d t}{t-x} \\
& \quad \leqslant \sum_{i \in A(x, \delta)} \frac{1}{\frac{a_{i}+b_{i}}{2}-\frac{b_{i}-a_{i}}{4^{i}}-a_{i}} 2 \frac{b_{i}-a_{i}}{4^{i}} \leqslant \sum_{i \in A(x, \delta)} \frac{2}{4^{i-1}} .
\end{aligned}
$$

Аналогично

$$
\left|\int_{x-\delta}^{x} f(t) \frac{\sin n(t-x)}{t-x} d t\right| \leqslant \sum_{i \in B(x, \delta)} \frac{2}{4^{i-1}} .
$$

При этом, если $A(x, \delta)$ или $B(x, \delta)$ пусто, то соответствующие интегралы равны нулю.

Зададим $\epsilon>0$ и выберем номер $i_{0}$ таким образом, чтобы выполнялось неравенство

$$
\sum_{i \geqslant i_{0}} \frac{2}{4^{i-1}}<\frac{\epsilon}{4}
$$

Если $x \in E$ и $x$ не является концом смежного к $E$ интервала, то оба множества $A(x, \delta)$ и $B(x, \delta)$ бесконечны для любого $\delta>0$. При этом, число $\delta>0$ можно выбрать настолько малым, чтобы числа из $A(x, \delta)$ и $B(x, \delta)$ были не меньше $i_{0}$.

Если $x \in E$ и $x$ является, для определенности, правым концом смежного к $E$ интервала, то выберем $\delta>0$ таким образом, что $f(t)=0$ при $t \in(x-\delta, x)$ и минимальный номер в $A(x, \delta)$ не меньше $i_{0}$.

Как известно (см. [1, гл. I, § 33]), для частичных сумм ряда Фурье для произвольного $\delta>0$ справедлива формула

$$
S_{n}(f, x)=\frac{1}{\pi} \int_{x-\delta}^{x+\delta} f(t) \frac{\sin n(t-x)}{t-x} d t+o(1),
$$

где $o(1)$ стремится к нулю при $n \rightarrow \infty$ равномерно по $x$. Отсюда следует, что для $x \in E$ и выбранного указанным образом по $\epsilon$ и $i_{0}$ числа $\delta>0$ можно найти такой номер $N$, что ввиду оценок (3)-(5) получим

$$
\left|S_{n}(f, x)\right|<\epsilon \quad \text { при } n \geqslant N .
$$

Следовательно, ряд Фурье $S(f)$ функции $f$ сходится к нулю в каждой точке множества $E$. Тем самьп мы получили, что $S(f)$ всюду сходится к $f$. Пример построен.

При построении примера выяснилось также, что в нем, в качестве функции $f$ не может фигурировать характеристическая функция какого-либо подмножества из $[0,2 \pi)$. А именно, справедлива 
ТЕорема 1. Если тригонометрический ряд сходится всюду или сходится всюду на интервале $(\alpha, \beta) \kappa$ функции $f$, то число значений функиии $f$ на $[0,2 \pi)$ или на $(\alpha, \beta)$, соответственно, не равно двум.

Вообще число значений суммы тригонометрического ряда, там где ряд сходится, может быть конечньм, счетным или континуальным, так как сумма ряда - борелевская функция на его области сходимости. Теорема 1 является следствием более общей теоремы.

ТЕорема 2. Пусть тригонометрический ряд (1) с условием (2) суммируется методом Римана всюду или всюду на интервале $(\alpha, \beta) \kappa$ функиии $f$. Тогда число значений функиии $f$ на $[0,2 \pi)$ или на $(\alpha, \beta)$, соответственно, не равно двум.

ДоКАЗАТЕЛЬСтво. Допустим, что ряд $S(1)$ с условием (2) суммируется методом Римана всюду на $[0,2 \pi)$ к характеристической функции $\chi_{E}(x)$, множества $E \subset[0,2 \pi)$, где $E \neq \varnothing$ и $E \neq$ $[0,2 \pi)$. Тогда, как следует из теоремы дю Буа-Реймона-Лебега ряд $S$ есть ряд Фурье $S\left(\chi_{E}\right)$ функции $\chi_{E}$. Функция Римана $F(x)$ ряда $S$, являющаяся суммой дважды почленно проинтегрированного ряда $S$, равна в этом случае

$$
F(x)=A x+B+\int_{0}^{x} d t \int_{0}^{t} \chi_{E}(u) d u .
$$

Суммируемость ряда (1) с условием (2) методом Римана означает, что

$$
D^{2} F(x)=\lim _{h \rightarrow 0} \frac{\Delta_{h}^{2} F(x)}{h^{2}}=\chi_{E}(x), \quad x \in[0,2 \pi),
$$

где $\Delta_{h}^{2} F(x)=F(x+h)+F(x-h)-2 F(x)$.

Учитьвая (6) получим, что

$$
D^{2} F(x)=\lim _{h \rightarrow 0} \frac{1}{h^{2}} \int_{0}^{h} m(E \cap(x+t-h, x+t)) d t,
$$

где $m(A)$ - мера множества $A$. Отсюда, в частности, следует, что $\chi_{E}-$ функция первого класса Бэра, а, значит, в силу теоремы Лебега-Хаусдорфа (см. $[7, \S 31, \mathrm{IX}])$ - борелевская функция первого класса. Следовательно, $[7, \S 31, \mathrm{I}], E-$ множество типа $G_{\delta}$ и $F_{\sigma}$ одновременно. Тогда и множество $E_{1}=[0,2 \pi) \backslash E$ имеет тип $G_{\delta}$ и $F_{\sigma}$.

Покажем, что если $E \cap(a, b) \neq \varnothing$, то найдется непустой интервал $\left(a_{1}, b_{1}\right) \subset E \cap(a, b)$. Действительно, множество $E \cap(a, b)$ имеет тип $F_{\sigma}$, поэтому $E \cap(a, b)=\bigcup_{k=1}^{\infty} F_{k}$, где $F_{k}$ - замкнутые множества. Так как $E \cap(a, b)$ имеет также тип $G_{\delta}$, в силу теоремы Бэра о категории хотя бы одно множество $F_{m}$ плотно в $E \cap\left(a_{1}, b_{1}\right) \neq \varnothing$ для некоторого интервала $\left(a_{1}, b_{1}\right) \subset(a, b)$. Следовательно, $E \cap\left(a_{1}, b_{1}\right)=F_{m} \cap\left(a_{1}, b_{1}\right)$. Допустим, что $\left(a_{1}, b_{1}\right) \backslash F_{m} \neq \varnothing$. Тогда найдется непустой смежный интервал $(c, d)$ к множеству $F_{m}$ на $\left(a_{1}, b_{1}\right)$. Пусть, для определенности, $c \neq a_{1}$, $c \in F_{m}$. Тогда $D^{2} F(c)=1$, так как $c \in F_{m}$, но с другой стороны $1 / h^{2} \Delta_{h}^{2} F(c) \leqslant 1 / 2$, так как $m(E \cap(c+t-h, c+t))=m(E \cap(c+t-h, c)) \leqslant h-t$ для малых значений $h$ и в силу формулы $(7)$. Полученное противоречие доказьвает, что $\left(a_{1}, b_{1}\right) \subset E \cap(a, b)$.

Аналогично, если $E_{1} \cap(c, d) \neq \varnothing$, то найдется непустой интервал $\left(c_{1}, d_{1}\right) \subset E_{1} \cap(c, d)$.

Обозначим через $G$ - внутренность множества $E$, а через $G_{1}$ - внутренность множества $E_{1}$. Заметим, что если $(a, b)$ - составляющий интервал $G$, то $a, b \in E$ и, значит, $D^{2} F(a)=D^{2} F(b)=$ 1. Аналогично, если $(c, d)$ - составляющий интервал множества $G_{1}$, то $c, d \in E_{1}$ и $D^{2} F(c)=$ $D^{2} F(d)=0$.

Пусть $\left(a_{1}, b_{1}\right)$ - составляющий интервал $G$. Тогда $D^{2} F\left(b_{1}\right)=1$. Выберем $h_{1}$ таким, образом, чтобы выполнялись неравенства $0<h_{1}<1, b_{1}-h_{1}<a_{1}$ и

$$
\Delta_{h}^{2} F\left(b_{1}\right) \geqslant \frac{31}{32} h^{2} \quad \text { для } h \leqslant h_{1} .
$$

Так как $b_{1}$ - конец составляющего интервала множества $G$, то $\left(b_{1}, b_{1}+h_{1}\right) \cap E_{1} \neq \varnothing$ и найдется интервал $(c, d) \subset\left(b_{1}, b_{1}+h_{1}\right) \cap E_{1}$. Пусть $\left(c_{1}, d_{1}\right)-$ составляющий интервал $G_{1}$, содержащий 
$(c, d)$. Тогда $b_{1}<c_{1}<b_{1}+h_{1}$. Выберем $t_{1}$ таким образом, чтобы $t_{1}<1 / 2 h_{1}, b_{1}<c_{1}-t_{1}$, $c_{1}+t_{1}<d_{1}$ и

$$
\Delta_{h}^{2} F\left(c_{1}\right) \geqslant \frac{1}{32} h^{2} \quad \text { для } h \leqslant t_{1} .
$$

Таким образом, $\left[c_{1}-t_{1}, c_{1}\right] \subset\left(b_{1}, b_{1}+h_{1}\right)$.

Продолжая построение, получим вложенную последовательность отрезков

$$
\left[c_{n}-t_{n}, c_{n}\right] \subset\left(b_{n}, b_{n}+h_{n}\right), \quad\left[b_{n+1}, b_{n+1}+h_{n+1}\right] \subset\left(c_{n}-t_{n}, c_{n}\right),
$$

где $t_{n+1}<\frac{1}{2} h_{n+1}<\frac{1}{2} t_{n}<1, n \in \mathbb{N}$, такую, что

$$
\begin{array}{ll}
b_{n} \in E, \quad & c_{n} \in E_{1}, \quad D^{2} F\left(b_{n}\right)=1, \quad D^{2} F\left(c_{n}\right)=0, \\
\Delta_{h}^{2} F\left(b_{n}\right) \geqslant \frac{31}{32} h^{2} \quad \text { для } h \leqslant h_{n}, \\
\Delta_{h}^{2} F\left(c_{n}\right) \geqslant \frac{1}{32} h^{2} \quad \text { для } h \leqslant t_{n} .
\end{array}
$$

Положим $\{d\}=\bigcap_{n=1}^{\infty}\left[c_{n}-t_{n}, c_{n}\right]$. Докажем, что $D^{2} F(d)$ не может равняться ни 0 , ни 1. Покажем, что из (9) следует, что $D^{2} F(d) \neq 0$. Заметим сначала, что

$$
m\left(E \cap\left(b_{n}, b_{n}+h_{n}\right)\right) \geqslant \frac{3}{4} h_{n} \quad \text { для } n \in \mathbb{N} .
$$

Допустим, что это не так. Тогда для некоторого $n$

$$
\begin{aligned}
\Delta_{h_{n}}^{2} F\left(b_{n}\right)= & \int_{0}^{h_{n}} m\left(E \cap\left(b_{n}+t-h_{n}, b_{n}+t\right)\right) d t=\int_{0}^{h_{n}} m\left(E \cap\left(b_{n}+t-h_{n}, b_{n}\right)\right) d t \\
& +\int_{0}^{h_{n}} m\left(E \cap\left(b_{n}, b_{n}+t\right)\right) d t \\
< & \int_{0}^{h_{n}}\left(t-h_{n}\right) d t+\int_{0}^{3 / 4 h_{n}} t d t+\int_{3 / 4 h_{n}}^{h_{n}} \frac{3}{4} h_{n} d t<\frac{31}{32} h_{n}{ }^{2} .
\end{aligned}
$$

Следовательно, неравенство (11) верно и из него следует, что

$$
\begin{aligned}
\Delta_{h_{n}}^{2} F(d)= & \int_{0}^{h_{n}} m\left(E \cap\left(d+t-h_{n}, d+t\right)\right) d t=\int_{0}^{h_{n}} m\left(E \cap\left(d+t-h_{n}, d\right)\right) d t \\
& +\int_{0}^{h_{n}} m(E \cap(d, d+t)) d t \geqslant \int_{0}^{3 / 4 h_{n}}\left(h_{n}-t-\frac{h_{n}}{4}\right) d t=\frac{9}{32} h_{n}{ }^{2}
\end{aligned}
$$

для $n \in \mathbb{N}$. Следовательно, $D^{2} F(d) \neq 0$. Аналогично с помошью (10) доказывается, что $D^{2} F(d) \neq 1$. Полученное противоречие доказьвает, что сумма тригонометрического ряда не может принимать двух значений, если ряд всюду сходится. Отсюда с помощью принципа локализации Римана доказывается и случай сходимости на интервале. Теорема доказана.

Отметим, что любое другое конечное число значений реализуется.

\section{СПИСОК ЦИТИРОВАННОЙ ЛИТЕРАТУРЫ}

1. Бари Н. К. Тригонометрические ряды. М.: Физматгиз, 1961. 2. Du Bois-Reymond. // Abh. Akad. Wiss. 1876. V. XII. P. 117-166. 3. Lebesgue H. // L’École norm. 1903. V. 20. № 3. P. 453-485. 4. Зигмунд А. Тригонометрические ряды. Т. I, II. М.: Мир, 1965. 5. Lebesgue H. Leçon sur les séries trigonométriques. Paris, 1906. 6. Tonelli L. Serie trigonometrische. Bologna, 1928. 7. Куратовский К. Топология. Т. І. М.: Мир, 1966. 